Carl, M., Kent, R., Levy, E. S., \& Whalen, D. (2020). Vowel acoustics and speech intelligibility in young adults with Down Syndrome. Journal of Speech, Language, and Hearing Research, 63, 674-687. https://doi.org/10.1044/2019_JSLHR-19-00204

Accepted for publication: Nov. 28, 2019

\title{
Vowel Acoustics and Speech Intelligibility in Young Adults with Down Syndrome
}

\author{
Micalle Carl ${ }^{\mathrm{a}}$, Raymond D. Kent ${ }^{\mathrm{b}}$, Erika S. Levyc, D. H. Whalen ${ }^{\mathrm{a}, \mathrm{d}, \mathrm{e}}$ \\ aProgram in Speech-Language-Hearing Sciences, City University of New York Graduate Center, \\ New York \\ ${ }^{b}$ Waisman Center, University of Wisconsin-Madison \\ ${ }^{\mathrm{c}}$ Teachers College, Columbia University, New York \\ ${ }^{\mathrm{d}}$ Haskins Laboratories, New Haven, CT \\ 'Yale University, New Haven, CT
}

Correspondence concerning this article should be addressed to Micalle Carl: mcarl@gradcenter.cuny.edu

Disclosure: The authors have declared that no competing interests existed at the time of publication.

\begin{abstract}
Purpose: Speech production deficits and reduced intelligibility are frequently noted in individuals with Down syndrome (DS) and are attributed to a combination of several factors. This study reports acoustic data on vowel production in young adults with Down syndrome, and relates these findings to perceptual analysis of speech intelligibility.

Method: Participants were eight young adults with DS, as well as eight age- and gender-matched typically developing (TD) controls. Several different acoustic measures of vowel centralization and variability were applied to tokens of corner vowels $(/ \mathrm{a} /, / \mathfrak{a} /, / \mathrm{i} /, / \mathrm{u} /)$ produced in common English words. Intelligibility was assessed for single-word productions of speakers with DS, by means of transcriptions from 14 adult listeners.

Results: Group differentiation was found for some, but not all, of the acoustic measures. Low vowels were more acoustically centralized and variable in speakers with DS than TD controls. Acoustic findings were associated with overall intelligibility scores. Vowel Formant Dispersion was the most sensitive measure in distinguishing DS and TD formant data.

Conclusion: Corner vowels are differentially affected in speakers with DS. The acoustic characterization of vowel production and its association with speech intelligibility scores within the DS group support the conclusion of motor control deficits in the overall speech impairment. Implications are discussed for effective treatment planning.
\end{abstract}




\section{Vowel Acoustics and Speech Intelligibility in Young Adults with Down Syndrome}

Down syndrome (DS) is one of the most common congenital, chromosomal disorders (Mai et al., 2013), and is associated with cognitive, physiological, and speech-language deficits (e.g., Kent \& Vorperian, 2013). Most individuals with DS demonstrate at least some degree of reduced speech intelligibility across the lifespan (Kumin, 1994; Wild, Vorperian, Kent, Bolt, \& Austin, 2018). Intelligibility reductions have been attributed to a number of possible contributing factors, including phonological, anatomical, sensory, and motor control differences, or any combination thereof. Although individuals with DS exhibit great phenotypic variation across physiological and developmental domains (Kent \& Vorperian, 2013), common characteristics include craniofacial and muscular differences from typically developing individuals, such as a reduced jaw size (Moura et al., 2008), an average tongue size within a smaller oral cavity, a high-arched palate, and muscular hypotonia of the general body and of oral/facial structures (Desai, 1997; Sforza, Dellavia, Allievi, Tommasi, \& Ferrario, 2012). Individuals with DS also frequently demonstrate impairment to auditory structures and functioning (Bernardi, Pires, Oliveira, \& Nisihara, 2017).

Despite the potential contribution of structural anomalies to differences in speech production, many researchers maintain that these do not fully account for the impairment in speakers with DS. Some have characterized the speech production deficit in this population as consistent with the specific motor speech disorders of dysarthria (Jones et al., 2019; Mahler \& Jones, 2012; Wilson, Abbeduto, Camarata, \& Shriberg, 2019), apraxia (Kumin, 2006; Wilson et al., 2019) or both (Rupela, Velleman, \& Andrianopoulos, 2016; Wilson et al., 2019), based on perceptual studies of the speech characteristics in DS.

\section{Vowel Acoustics and Intelligibility}

Vowels have long been described and measured within the acoustic formant space, from which articulation is inferred (e.g., Chiba \& Kajiyama, 1941). Variation of vowel formants within the first and second formant frequency $(\mathrm{F} 1 \mathrm{xF} 2)$ acoustic space reflects the primary features of vowels, namely tongue height and advancement (Peterson \& Barney, 1952). To the extent that the acoustic values correspond to these articulatory dimensions, the maximal acoustic values of the corner vowels (e.g., /a/, /æ/, /i/, /u/ in American English) represent the most extreme tongue positions for vowel production (e.g., Neel, 2008). Conversely, a reduction of the space enclosed by the four corner vowels is presumed to indicate decreased articulatory movement of the tongue toward the vowel targets, and is a commonly reported characteristic of dysarthria of varying etiologies, in both children (Hustad, Gorton, \& Lee, 2010; Levy et al., 2016; Levy, Chang, Ancelle, \& McAuliffe, 2017; Moura et al., 2008) and adults (e.g., Kent \& Kim, 2003; Lansford \& Liss, 2014b; Skodda, Visser, \& Schlegel, 2011). Although several metrics can be used to calculate and describe acoustic space and/or its centralization, the combined application of metrics may be most effective in analyzing formant data, and thus the associated articulation, by providing complementary information (Kent \& Vorperian, 2018).

Frequently measured by the area enclosed by the corner vowels in the acoustic space (vowel space area, or VSA), the degree of vowel centralization often serves as an index of dysarthria severity, as indicated by correlated measures of intellegibility (Yunusova, Weismer, Kent, \& Rusche, 2005). However, despite the findings of correlation between vowel space area and speech intelligibility, several studies have reported a lack of sensitivity of this acoustic metric in distinguishing between clinical and typical populations (Sapir, Ramig, Spielman, \& Fox, 2010; Tjaden \& Wilding, 2005; Weismer, Jeng, Laures, Kent, \& Kent, 2001). This has been attributed, 
in part, to high sensitivity of the VSA metric to inter-speaker differences (Sapir et al., 2010), as well as to its calculation from vowel formant means, as opposed to individual token values (Karlsson \& van Doorn, 2012b). In light of its varying effectiveness, several alternative measures to the VSA have been suggested and implemented with some success. For investigations of the vowel acoustic space with the corner vowels alone, the Formant Centralization Ratio (FCR; Sapir et al., 2010) and the Vowel Formant Dispersion (VFD; Karlsson \& van Doorn, 2012a, 2012b) metrics have both demonstrated increased sensitivity in distinguishing speakers with speech disorders and typically developing (TD) controls. The VFD metric has specific applicability to vowel centralization, and not group differentiation alone, as it provides a measure of degree of centralization, as well as the direction (i.e., which of the corner vowels) from which the centralization occurs (Karlsson \& van Doorn, 2012b). In a novel analysis created for speakers with DS, termed the DS Vocalic Anatomical Functional Ratio (DS-VR), Moura et al. (2008) calculated the ratio of $\mathrm{F} 2$ values of $/ \mathrm{i} /$ and $/ \mathrm{u} /$. This metric was shown to be a sensitive indicator of group differences between children with DS and their typical peers (Moura et al., 2008). The authors attribute this group differentiation, at least partially, to the anatomical and/or physiological factors of limited tongue movement in a smaller than average pharyngeal cavity in the speakers with DS, resulting in higher $\mathrm{F} 2 / \mathrm{u} /$ values than those of typical controls.

Recent literature has demonstrated that calculation of individual vowel cluster distribution within the acoustic space is another means of relating vowel acoustics to speech intelligibility (Kent \& Vorperian, 2018; McCloy, Wright, \& Souza, 2014). Increased stability and consistency of vowel production (and thus tighter vowel clusters) is associated with increased articulatory proficiency and speech motor control (e.g., Yang \& Fox, 2013). Thus, a larger vowel target area and increased variability of associated acoustic features may be indicative of reduced maturation and/or proficiency of the speech motor system. Measures of vowel cluster spread and/or overlap in non-corner (e.g., central) and corner vowels have also been correlated with speech intelligibility in both healthy speakers (McCloy et al., 2014) and speakers with dysarthria due to various neurodegenerative (Lansford \& Liss, 2014a) or neuromotor (Connaghan \& Patel, 2017; Kim, Hasegawa-Johnson, \& Perlman, 2011) disorders.

Although acoustic features of vowel production are frequently correlated with intelligibility scores in studies of disordered speech production, there are mixed positions regarding the nature of this acoustic deficit, namely whether it is merely an index of intelligibility severity or is instead a contributing factor to the decreased intelligibility. Some studies suggest that acoustic measures may be strong indices of intelligibility severity in dysarthric speakers, but not necessarily integral components of the intelligibility deficit (Yunusova et al., 2005). In contrast, others have interpreted findings of strong correlations between vowel acoustic measures and perceptual vowel accuracy and/or intelligibility scores, as signifying a strong contribution of the impaired vowels to the perceptual deficit, and thus indicating a causal relationship between vowel acoustics and perception (Lansford \& Liss, 2014a).

\section{Vowel Acoustics and Speech Intelligibility in DS}

Characterization of vowel production in speakers with DS has been demonstrated with emerging evidence on both the acoustic and perceptual levels, with some relation to overall intelligibility. Moura et al. (2008) reported centralization of vowel space in children with DS as well as group differentiation for the DS-VR metric between the DS and TD groups. Bunton and Leddy (2011) studied x-ray microbeam data in two male adults with DS. They found a relatively 
centralized acoustic vowel space, as well as a generally reduced articulatory working space in xray microbeam data, in the adults with DS, in comparison to age-matched controls.

Impairment of high-vowel production may indicate anatomical influence on vowel acoustics, in light of the impact of palatal morphology on tongue shape during production of the high front vowel (Gick, Allen, Roewer-Després, \& Stavness, 2017; Lammert, Proctor, \& Narayanan, 2013), as well as the potentially smaller posterior palate size in individuals with DS (as suggested by Moura et al., 2008). In contrast, differences to vowel space area or dispersion would likely indicate a motor speech component, as seen among speakers with dysarthria (e.g., Kent \& Kim, 2003; Levy et al., 2017).

Intelligibility analyses for individuals with DS have also demonstrated the contribution of vowel errors to the overall intelligibility impairment. Bunton, Leddy, and Miller (2009) tested 19 phonemic contrasts in adults with DS and reported vowel contrast errors among the most frequently occurring errors (e.g., long vs. short vowel, high vs. low vowel, front vs. back vowel). Frequent consonant contrast errors included initial and final cluster vs. singleton, initial glottal (/h/) vs. no consonant, and fricative place of production. Finally, Wild et al. (2018) reported a contribution of reduced vowel intelligibility to the overall accuracy of single-word productions in individuals with DS across the age-span of children to adults. Conclusions from these acoustic and perceptual studies indicate that the speech impairment in DS may be associated with motor deficits in the tongue and related structures, and not structural anomalies alone. Specifically, while structural difference may account partially for errors related to consonant articulation, including errors relating to place of articulation of stops and fricatives (Timmins, Cleland, Wood, Hardcastle, \& Wishart, 2009), these and other errors (e.g., vowel errors) may also imply motor control deficits for positioning of the articulators within the oral cavity (Bunton et al., 2009). However, none of the studies related measures of acoustic space with intelligibility scores directly. Such information of acoustic-intelligibility associations would provide valuable information regarding both the nature of the speech deficit and the impact vowel production has on overall intelligibility in speakers with DS.

\section{Purpose}

The purpose of this study was to describe the acoustic characteristics of vowel production in young adults with DS, and to relate these data to intelligibility scores. This information would facilitate an increased understanding of the general nature of the speech disorder in speakers with DS (e.g., anatomical, phonemic, motor). Specific research questions are listed as follows:

1. Do speakers with DS exhibit a reduction in the vowel acoustic space and/or increased acoustic variability of vowel production in comparison to TD peers?

2. Is single-word intelligibility correlated with acoustic vowel space in speakers with DS?

\section{Method}

\section{Part 1: Acoustic Analysis}

\section{Participants}

A total of 16 young adults participated in the study, 8 with Down syndrome (DS) and 8 healthy, typically developing (TD) young adults. All participants were between 19 and 27 years of age; mean ages were 21 years of age for the DS group, and 22 years for the TD group. Gender was matched between the two groups, with 5 males and 3 females in each. Criteria for participants with DS also included report of a general cognitive level adequate for tolerance of the experimental design, as relayed by a parent or caretaker. TD participants reported no history of cognitive, 
language, or hearing impairment, as well as no persisting speech or articulation disorder. All participants used American English as their primary language, as reported by self or parent/caretaker. A history of pressure-equalization (PE) tubes was reported for 5 out of 8 participants with DS, although none of the participants reported any significant hearing loss, and none wore hearing amplification devices. Mild dysfluency/stuttering-like behaviors were noted in the speech of two participants (DSF8, DSM10). The participants with DS presented with a wide range of single-word intelligibility scores, as detailed in the Intelligibility section below.

All participants took part in a pure tone hearing screening prior to the experimental procedures. The hearing screening was conducted in a sound-treated booth, with presentation of tones at $25 \mathrm{~dB} \mathrm{HL}$ for $500,1,000,2,000,4,000 \mathrm{~Hz}$, bilaterally. Of the participants with DS, a total of 4 participants passed the screening, and another 2 participants passed for all frequencies except one from a single ear. Two participants with DS did not pass the hearing screening at most frequency presentations (DSF5, DSM10), although reports from their parent or guardian indicated no significant concerns regarding hearing acuity. Due to the frequent presence of middle ear fluid or other hearing impairment in individuals with DS, participants with DS were not excluded from the current study if they did not pass the presented hearing screening (as in Wild, Vorperian, Kent, Bolt, \& Austin, 2017). All TD participants passed the hearing screening. All procedures of this study received ethical approval from the Institutional Review Board at The Graduate Center, CUNY.

\section{Stimuli}

Experimental stimuli were single-syllable words containing one of the 4 corner vowels $(/ \mathrm{a} /$,

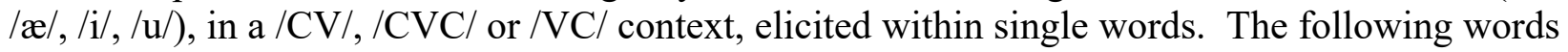
were used: /a/: hop, pot, hot, mop; /æ/: app, hat, bat, map; /i/: heap, bee, eat, feet, beep; /u/: hoop, hoot, food, boot, boo. The choice of words was guided by the need to ensure that most stimuli were likely to be within the linguistic repertoire of individuals with language and/or cognitive deficits and could be represented by pictures. Although all the participants with DS had a reading level of at least basic words, reading skill was not a criterion for inclusion within the study. Adjacent consonants were limited, to the extent possible, to non-lingual consonants, in order to avoid lingual coarticulatory effects during the vowel production (Strange et al., 2007). Nonetheless, some lingual consonants were included in the word stimuli in order to allow for variation of words and thus limit the monotony of word elicitation. Lingual consonants were limited to final position of words, as lingual consonants in this position are noted to have a reduced impact on vowel formant values compared to those in initial position of the syllable (Hillenbrand, Clark, \& Nearey, 2001). Following initial familiarization with the stimuli prior to the experimental procedures, the participants were presented with stimuli orthographically and pictorially. Each stimulus was presented to the participant approximately 10 times, with all stimuli randomized during presentation.

\section{Procedures}

Acoustic data were collected as part of a larger study, in conjunction with ultrasound recording (Carl, 2018). The stimuli were recorded with a Sennheiser ME66/K6 Combo Shotgun Mic System microphone, placed next to the ultrasound stand, at an approximately $30-45^{\circ}$ angle and approximately $10 \mathrm{~cm}$. from the speaker's mouth. The acoustic signal was sent to a Rane MLM82 mic/line Mixer, which also functioned as a preamp to the acoustic signal, and was digitized at $44100 \mathrm{~Hz}$. Acoustic stimuli were collected concurrently during ultrasound recording, in order to allow for subsequent correlation between acoustic and ultrasound data. The ultrasound 
setup included an ultrasound probe attached to a spring-loaded stand. As such, the probe placed under the participant's jaw moved in conjunction with the jaw, thus allowing freer movement of the jaw than in fixed-distance (helmet-based) systems.

For each participant, a total of 33-34 tokens per vowel, on average, was used for analyses. It should be noted that for two participants in each speaker group (DSM3, DSF5, TDF2, TDM5), /i/ and/or /u/ tokens from the CV stimuli "boo" and "bee," respectively, were removed from the analyses, due to unusable concurrent ultrasound data. Stimuli were not excluded from analyses based on perceptual accuracy of the token.

Vowel formants were calculated in Praat, version 6.0.28 (Boersma \& Weenink, 2017). Values of F1, F2, and F3 were recorded at the midpoint of the vowel (e.g., (Adank, Smits, \& van Hout, 2004; Tjaden, Lam, \& Wilding, 2013) using Linear Predictive Coding (LPC) analysis, by means of a Praat-coded script. Further settings for formant calculation included maximum formant value of $5500 \mathrm{~Hz}$, an analysis window length of 0.025 seconds, and a time step between two consecutive analysis frames of 0.01 seconds. The maximum number of formants was set to 5 in most cases. However, due to frequent errors in LPC estimates, formant trajectories generated by LPC for each individual trial, overlaid on the spectrogram, were visually inspected prior to recording the formant values. When a discrepancy between the predicted formant and spectrographic display was noted (e.g., the LPC tracks were off the dark formant regions as visualized on the spectrogram), the maximum formant value on the script was adjusted to 4 or 6 maximum formants, in order to achieve agreement between LPC formant tracks and the formants visualized on the spectrogram. All other script settings remaining the same, and the new formant values obtained from the change in maximum formant values were used in place of the originals. This occurred on approximately $20 \%$ of the total number of stimuli. All mathematical and statistical calculations related to formant analyses were conducted in R (R Core Team, 2017).

\section{Reliability}

In order to confirm the accuracy of formant tracking and estimation, both intra- and interrater reliability analyses were conducted on $20 \%$ of the data (432 tokens), in which tokens were reanalyzed by the first author (intra-rater) as well as by an outside judge. The reliability analyses were conducted from the point of vowel boundary marking on the spectrogram through formant estimation. Results of the intra-rater reliability testing demonstrated a strong positive correlation between results, with very high Pearson product-moment correlation coefficients for both F1 and F2 values, across all vowels $(r=.97-.99$ across vowels, $p<.001)$. Results of the inter-rater reliability testing also demonstrated strong, positive correlations between the original and reanalyzed data. Prior to calculation of the correlations, a total of four outlier/error values for /i/ (F2) and $/ \mathrm{u} /$ (F1) were noted on the correlation plots and removed from the calculations. Subsequently, Pearson product-moment correlations were calculated for both F1 and F2 values across vowels. Results revealed strong positive correlations for $/ \mathfrak{x} /$ and $/ \mathrm{u} /$ vowels, for both $\mathrm{F} 1$ and F2 $(r=.97-.99, p<.001)$, as well as positive correlations for $/ \mathrm{a} /$ and $/ \mathrm{i} /$ vowels, for both F1 and F2 $(r=.92-.99, p<.001)$.

\section{Formant Normalization}

Normalization of the formant values was conducted for certain analyses in order to reduce inter-speaker variability caused by anatomic or physiological differences in the speaker's vocal tract, including the effect of gender on the formant values (Adank et al., 2004; Vorperian \& Kent, 2014). Lobanov's z-score normalization (Lobanov, 1971) was used for the current acoustic 
analysis. The calculation of Lobanov's z-score normalization applied the following calculation (Equation 1) to the acoustic data, separately for each speaker and for each of the formants (F1, F2):

\section{Equation 1}

$F_{s i}^{\text {normalized }}=\frac{F_{s i}-\mu_{s i}}{\sigma_{s i}}$, where $s=$ individual speaker, and $i=$ formant number $(\mathrm{F} 1, \mathrm{~F} 2)$.

\section{Vowel Space Metrics}

Several different metrics were applied to the acoustic data, to determine the presence of group differences and describe the acoustic characterization of vowel production in both speaker groups. These metrics included: Vowel Space Area (VSA; Vorperian \& Kent, 2014), the Formant Centralization Ratio (FCR; Sapir, Ramig, Spielman, \& Fox, 2010), the DS Vocalic Anatomical Functional Ratio (DS-VR; Moura et al., 2008), the Vowel Formant Dispersion (VFD; Karlsson \& van Doorn, 2012), and ellipse areas of vowel clusters (McCloy et al., 2014). Each of these is described in the following paragraphs.

\section{Vowel Space Area (VSA)}

The Vowel Space Area (VSA) is a general measure of the area enclosed by the corner vowels ([i æ $\mathrm{a} u]$ ) within the acoustic space. The VSA employs the equation for the area of an irregular parallelogram, using the mean value of each formant per vowel, separately for each speaker, as stated in the following Equation 2 (Vorperian \& Kent, 2014):

\section{Equation 2}

$$
\begin{aligned}
\mathrm{VSA}= & 0.5 *\left\{\left(\mathrm{~F}_{2} / \mathrm{i} / * \mathrm{~F}_{1} / \mathfrak{a} /+\mathrm{F}_{2} / \mathfrak{a} / * \mathrm{~F}_{1} / \mathrm{a} /+\mathrm{F}_{2} / \mathrm{a} / * \mathrm{~F}_{1} / \mathrm{u} /+\mathrm{F}_{2} / \mathrm{u} / * \mathrm{~F}_{1} / \mathrm{i} /\right)-\left(\mathrm{F}_{1} / \mathrm{i} / * \mathrm{~F}_{2} / \mathfrak{a} /+\mathrm{F}_{1} / \mathfrak{x} / *\right.\right. \\
& \left.\left.\mathrm{F}_{2} / \mathrm{a} /+\mathrm{F}_{1} / \mathrm{a} / * \mathrm{~F}_{2} / \mathrm{u} /+\mathrm{F}_{1} / \mathrm{u} / * \mathrm{~F}_{2} / \mathrm{i} /\right)\right\}
\end{aligned}
$$

\section{Formant Centralization Ratio (FCR)}

Similarly to the VSA, the Formant Centralization Ratio (FCR) is calculated from vowel means, for each of the vowel formants (F1, F2). However, in contrast the VSA, the FCR is a ratio of values, thus creating a normalization-like procedure and naturally minimizing variability between speakers with different vocal tract anatomy, including gender differences (Sapir et al., 2010). The calculation of the FCR is expressed as the following (Equation 3 ):

\section{Equation 3}

$\mathrm{FCR}=\left(\mathrm{F}_{2} / \mathrm{u} /+\mathrm{F}_{2} / \mathrm{a} /+\mathrm{F}_{1} / \mathrm{i} /+\mathrm{F}_{1} / \mathrm{u} /\right) /\left(\mathrm{F}_{2} / \mathrm{i} /+\mathrm{F}_{1} / \mathrm{a} /\right)$

In light of its robustness to differences in gender among speakers, FCR was calculated on the originally obtained $\mathrm{Hz}$ formant values without using the data normalization procedure.

\section{DS Vocalic Anatomical Functional Ratio (DS-VR)}

The DS-VR (Moura et al., 2008) also included mean values of the formants for each vowel, individually per speaker, and was calculated as follows (Equation 4):

\section{Equation 4}

$\mathrm{DS}-\mathrm{VR}=\frac{\mathrm{F} 2 / \mathrm{i} /}{\mathrm{F} 2 / \mathrm{u} /}$

Similarly to the FCR metric, the DS-VR metric was calculated only with Hz values, as the ratio of values provides an inherent normalization-like procedure.

\section{Vowel Formant Dispersion (VFD)}

A final metric of vowel space calculation, the Vowel Formant Dispersion (VFD) measure, calculates the distances of individual formant values in the F1xF2 space from a centroid within 
that space, thus relating the measure to the centralization noted within certain speech disorders (Karlsson \& van Doorn, 2012b). A distinguishing factor of the VFD metric in comparison to previously used metrics of vowel dispersion (e.g., Bradlow, Torretta, \& Pisoni, 1996) is the placement of the centroid for calculation of dispersions. In contrast to previous measures, in which the centroid is calculated as the mean of both F1 and F2 of all vowels in the acoustic F1xF2 space, the VFD relies on a weighted centroid, in which only certain values are included in its calculation. More specifically, the weighted centroid of the VFD metric includes the mean of all F1 values, while the F2 mean includes only F2 values of those tokens in which the F1 values are lower than the F1 mean (Equation 5, Equation 6).

\section{Equation 5}

\section{Equation 6}

$F_{1 s_{\text {mean }}}=\operatorname{mean}\left(F_{1 s}\right) ;$ where $s$ refers to each individual speaker

$F_{2 s_{\text {w.mean }}}=\operatorname{mean}\left(F_{2 s}\right)\left\{V\left(F_{2}, F_{1}\right): F_{1 s}<F_{1 s_{\text {mean }}}\right.$; where $s$ refers to each individual speaker, and $V$ refers to all vowels within analyses for the individual speaker.

As explained by Karlsson and van Doorn (2012b), the weighted mean is a more robust placement of the mean than direct averages of vowel formant values, and accomplishes two primary goals. First, it allows for comparison of vowel spaces that may differ in the inclusion of 3 versus 4 corner vowels. Second, the inclusion of only the specified F2 values into the centroid calculations allows for additional strength of its placement, as it excludes possible inefficient productions of front, open vowels.

For application of the VFD metric to the current data, a weighted centroid was computed separately for each speaker. To prevent a skewing of the weighted centroid towards any vowel with a large number of tokens, the centroid was calculated from means of vowels, per speaker. Thus, the $F 1$ value of the weighted centroid was calculated as an average of $F_{1} / \mathrm{a} /, \mathrm{F}_{1} / \mathfrak{x} /, \mathrm{F}_{1} / \mathrm{i} /$, and $\mathrm{F}_{1} / \mathrm{u} /$ means. The $\mathrm{F} 2$ value for the weighted centroid was calculated just from $\mathrm{F} 2$ values in which corresponding F1 values were below the $F 1$ mean, namely an average of $F_{2} / \mathrm{i} /$ and $F_{2} / \mathrm{u} /$ means. Once the weighted centroid was obtained, a vector was calculated to connect each point (F2, F1) within the speaker's acoustic space to the centroid, using the following distance equation (Equation 7):

\section{Equation 7}

$$
V F D_{s i}=\sqrt{\left(F 1_{s i}-F 1_{s_{\text {mean }}}\right)^{2}+\left(F 2_{s i}-F 2_{s_{\text {w.mean }}}\right)^{2}}
$$

where $s$ refers to each speaker, $i$ refers to each $(\mathrm{F} 2, \mathrm{~F} 1)$ vowel point token, and w.mean refers to the weighted centroid, as described above.

\section{Vowel Cluster Areas}

The size of the individual speaker vowel clusters in the acoustic formant space was calculated and used as a measure of within-speaker variability, as in McCloy et al. (2014), and thus also articulatory proficiency. Ellipses surrounding the individual vowel clusters were drawn at the $95 \%$ confidence interval, calculated using the phonR package in R (McCloy, 2016), in conjunction with an author-created script (McCloy, 2017). The ellipse of each vowel cluster was calculated from the mean of the formant values within the F1xF2 space, as well as the covariance between F1 and F2, in order to determine the direction and spread of the ellipse. 


\section{Statistical Analysis}

Quantitative analyses included two-tailed, two-sample $t$-tests, for certain metrics, while linear mixed effects (LME) modeling and analysis of variance (ANOVA) were applied to others, as detailed in the results section below.

Linear mixed effects model

Specifically for the VFD metric, in which all data tokens were considered within the analysis, linear mixed effects (LME) models were applied to the data, in order to determine whether the groups differed for results of the metric, and whether any difference was noted across vowels. This analysis was applied using the lme4 package (Bates, Maechler, Bolker, \& Walker, 2015) in R (R Core Team, 2017). The linear mixed effects model was chosen for the data analysis due to its consideration of both fixed and random effects as well as its relative strength in addressing missing or unbalanced data (Baayen, Davidson, \& Bates, 2008). All post-hoc analyses were conducted using the lsmeans package (Lenth, 2016) in R (R Core Team, 2017), in which least-square means, or adjusted prediction means, were obtained from the fitted LME model, and used for both comparisons (e.g., across and within group) and significance testing.

\section{Results}

A two-tailed, two-sample t-test was used to determine whether results of each of the following three metrics differed between the speaker groups: Vowel Space Area (VSA), Down Syndrome Anatomic Vocalic Ratio (DS-VR), and the Formant Centralization Ratio (FCR). For each metric analysis, a Levene's test using the $\mathrm{F}$ distribution was used to check whether the assumption of equality of variance for t-tests was met. Results indicated no significant group differences in variance for each of the three metrics: $\operatorname{VSA}(\mathrm{F}(7,7)=1.64, p=0.53), \mathrm{DS}-\mathrm{VR}(\mathrm{F}(7,7)$ $=.80 ; p=.78)$, and FCR $(\mathrm{F}(7,7)=.98, p=.98)$. Similarly, comparisons of group means for each of the metric outcomes revealed no significant differences, as follows: VSA $(t(14)=.38, p=.71)$, $\operatorname{DS}-\operatorname{VR}(t(14)=-1.79, p=.1), \operatorname{FCR}(t(14)=.27, p=.79)$. These data are summarized in Table 1.

\section{Table 1: Statistical Analyses of VSA, DS-VR, \& FCR metrics}

\begin{tabular}{lll}
\hline & Levene's test & t-test \\
\hline $\begin{array}{l}\text { Vowel Space Area (VSA) } \\
\text { Down Syndrome Anatomic-Vocalic }\end{array}$ & $\mathrm{F}(7,7)=1.64, p=.53$ & $t(14)=.38, p=.71$ \\
Ratio (DS-VR) & $\mathrm{F}(7,7)=.80, p=.78$ & $t(14)=-1.79, p=.1$ \\
Formant Centralization Ratio (FCR) & $\mathrm{F}(7,7)=.98, p=.98$ & $t(14)=.27, p=.79$ \\
\hline
\end{tabular}

\section{Vowel Formant Dispersion (VFD)}

Results of the LME model for the VFD results revealed a significant main effect of Group $(\beta=-0.19, \mathrm{SE}=0.04, t(25.80)=-5.04, p<.001)$, as well as for Vowel $/ \mathfrak{a} /(\beta=-.46, \mathrm{SE}=.06$, $t(15.30)=-7.23, p<.001)$, and Vowel $/ \mathrm{u} /(\beta=-.20, \mathrm{SE}=.06, t(16.00)=-3.29, p<.01)$. The interactions were also significant for Group $(D S) x$ Vowel $/ \mathrm{i} /(\beta=.22, \mathrm{SE}=.03, t(2127.30)=7.04$, $p<.001)$, and Group $(D S) x$ Vowel $/ \mathrm{u} /(\beta=0.25, \mathrm{SE}=.03, t(2127.20)=8.01, p<.001)$. However, due to the multi-level factors and significant interaction terms, all interpretation of LME results was conducted only from the post-hoc analyses listed below, in order to obtain estimates and 
significance of group comparisons. Degrees of freedom were calculated using the Satterthwaite method and confidence interval level was set at 0.95 . Results of the predicted means calculations are presented visually in Figure 1 below.

Figure 1: Comparison of predicted dispersion means (Ismeans) for each of the vowels, across groups

\section{Vowel Formant Dispersion: Least Square Means Comparison}

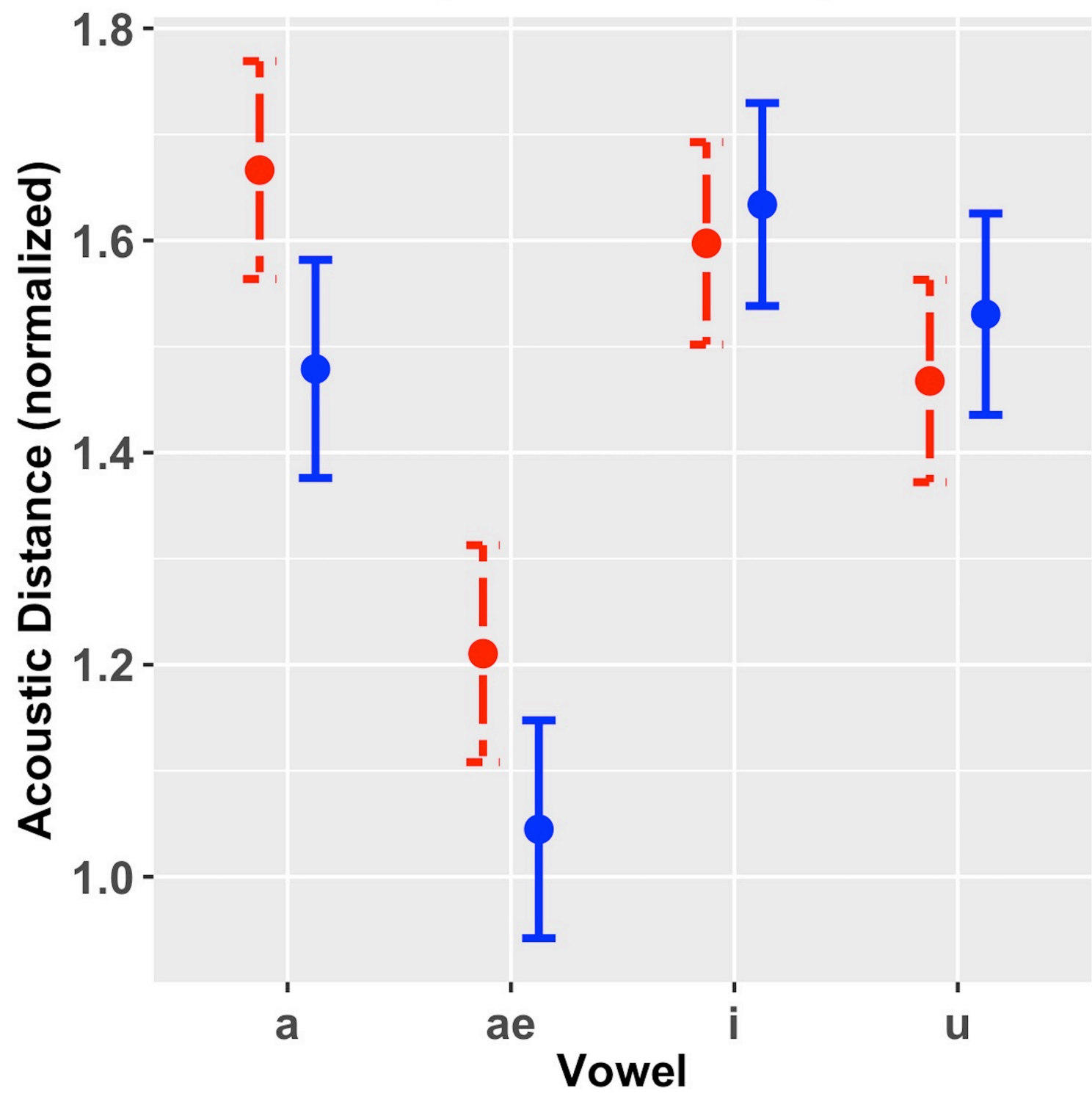

Group

- T- TD

$\vartheta$ DS 
The contrasts were calculated as t-tests within the lsmeans package, with the following obtained $p$-values (Table 2):

Table 2: Comparison of vowel dispersion results, across groups; starred contrasts are statistically significant

\begin{tabular}{llllll}
\hline $\begin{array}{l}\text { Contrast } \\
\text { TD-DS }\end{array}$ & Estimate & $S E$ & $d f$ & t.ratio & p.value \\
\hline Vowel $=a:$ & 0.187 & 0.037 & 25.660 & 5.014 & $*<.0001$ \\
Vowel $=\alpha e:$ & 0.166 & 0.037 & 24.680 & 4.471 & $* 0.0002$ \\
Vowel $=i:$ & -0.039 & 0.038 & 27.05 & -1.020 & 0.317 \\
Vowel $=u:$ & -0.064 & 0.037 & 25.72 & -1.697 & 0.102 \\
\hline
\end{tabular}

Results of individual contrasts demonstrate that the dispersion of the two groups differed significantly in low vowels $/ \mathrm{a} /(t(25.66)=5.014, p<.0001)$ and $/ æ /(t(24.68)=4.471, p=.0002)$, with TD speakers demonstrating greater dispersions of 0.19 normalized $\mathrm{Hz}$ and 0.17 normalized $\mathrm{Hz}$ from the centroid for $/ \mathrm{a} /$ and $/ \mathfrak{a} /$ respectively, than the speakers in the DS group. Group differences for the high vowels $/ \mathrm{i} /$ and /u/ were not significant.

\section{Vowel Cluster Areas}

A visualization of the ellipse areas is presented in the boxplots below (Figure 2).

Figure 2: Ellipse areas in normalized $\mathrm{Hz}^{2}$ values, by group

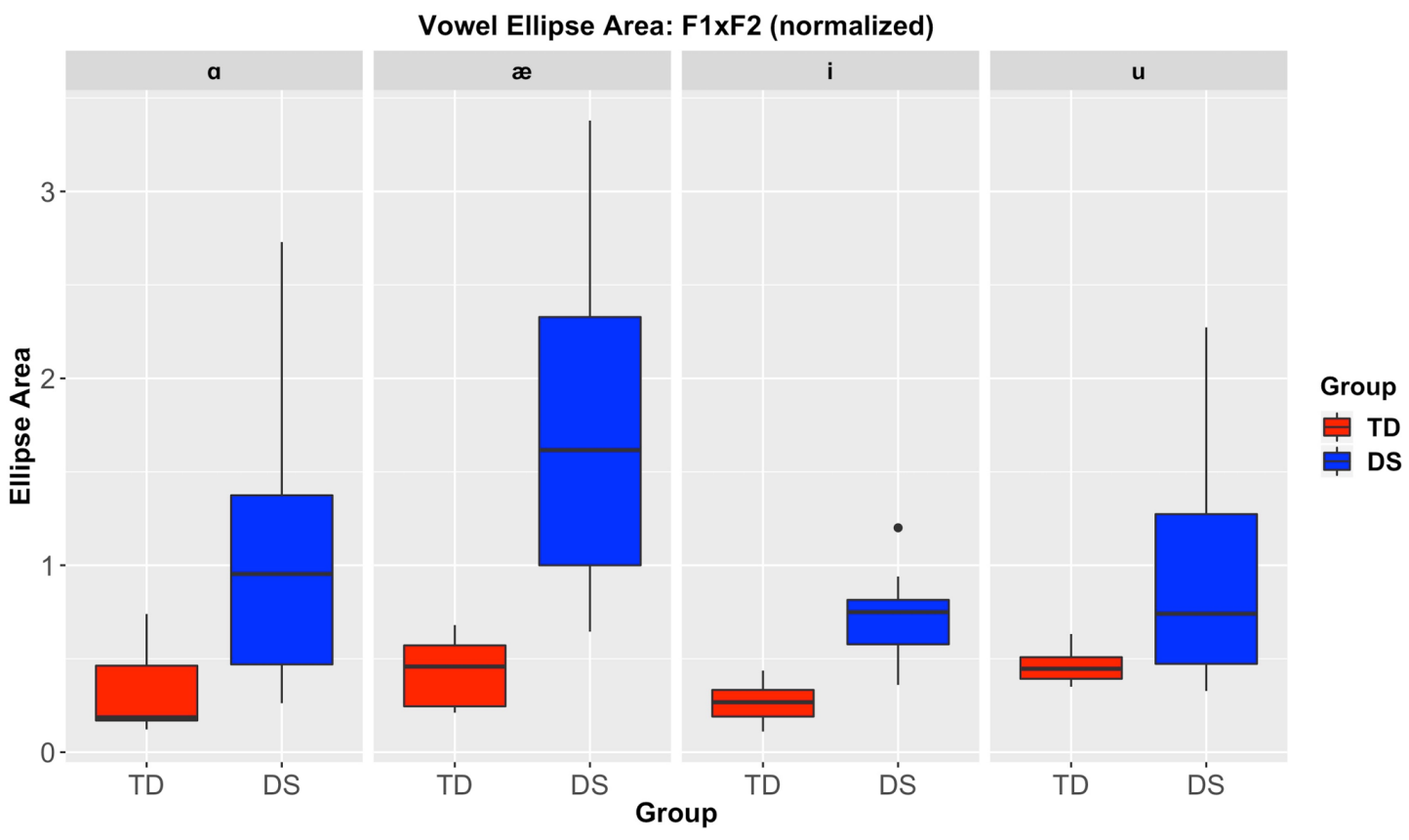


To test whether the groups differed in the size of the vowel clusters, as measured by the 95\% confidence ellipses, and whether any such difference varied by vowel, a two-way ANOVA was conducted. The ANOVA revealed significant main effects for both Group $[\mathrm{F}=31.292, p<$ $.001]$, and for Vowel [F $=3.08, p=.035]$, but no significant interaction between the two main effects. Tukey post-hoc comparisons were conducted for the main effect of Vowel, as well as for the interaction of Group by Vowel. Results of the post-hoc analyses for the factor of Vowel revealed a significant difference between means of only one vowel pair, the $/ \mathrm{i} /-/ \mathfrak{r} /$ vowel pair, in which the mean $/ \mathfrak{a} /$ cluster area was greater than $/ \mathrm{i} /$ cluster area by 0.59 normalized $\mathrm{Hz}^{2}(p=.020)$. Post-hoc analysis on the interaction term was conducted to determine whether significant differences were found for individual vowel contrasts, both across and within TD and DS groups. Statistically significant differences were noted for both the group comparison of $/ \mathfrak{a} /(\mathrm{DS} / \mathfrak{a} />$

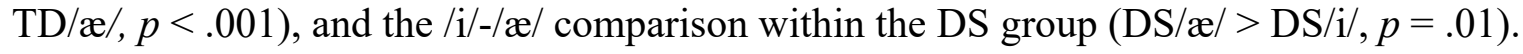

\section{Part 2:Intelligibility Testing}

\section{Method}

\section{Procedures}

Intelligibility testing and analysis were conducted only for productions by participants with DS. The intelligibility assessment used for the testing, the Test of Children's Speech (TOCS+; Hodge \& Gotzke, 2010; Hodge et al., 2009), is a computer-based assessment, in which participants' word and sentence-level productions are recorded and later presented to listeners, for scoring and analysis of intelligibility. As part of the recording, each participant is presented with a spoken production of a target word or sentence, accompanied by visual stimuli of both a picture and orthographic representation. The participant is then instructed to repeat the target. No reading skills are required. Although the speakers with DS in the current study were considerably older than the child speakers used for validation of this intelligibility assessment (Hodge \& Gotzke, 2014), the TOCS + was chosen in light of the potential cognitive deficits and limited reading abilities in the DS population. Recordings of the word and sentence-level stimuli were conducted separately from the acoustic recording sessions described earlier. Each participant was seated in a sound-treated sound booth, with a computer screen displaying the visual stimuli, and wore a head-mounted microphone (Audio-Technica AT8538 Power Module) to ensure a consistent mouth-to-microphone distance during recording. Word-level productions were elicited from one of three word-lists, all consisting of 78 words, and all balanced for a pre-determined selection of phonemic contrasts (Hodge \& Gotzke, 2014). Stimuli were randomized during presentation to the subject.

\section{Listeners}

A total of 14 healthy adult listeners participated in intelligibility judgments of single-word productions recorded from speakers with DS. None of these listeners were included in the control group of the acoustic analyses presented above. All listeners were between 19 and 48 years of age (mean age: 28 years). Of the listeners, 11 were female and 3 were male. All listeners self-reported their primary language to be English, as well as their having no significant history of cognitive, hearing, and/or speech-language impairment. Furthermore, they passed a hearing screening conducted in a sound-treated booth, with presentation of tones at 25dB HL for 500, 1,000, 2,000, $4,000 \mathrm{~Hz}$, bilaterally. Listeners reported no consistent exposure to individuals with DS. 
Listeners were seated in a quiet room in front of a Dell XPS 8900 computer (Intel ${ }^{\circledR}$ CoreTM Processor, i7-6700 CPU @3.40GHz; 16.0 GB RAM, 64-bit Operating System) on which the recorded stimuli were presented. As conducted in the original TOCS+ implementation (Hodge \& Gotzke, 2014), listeners were instructed to read through a word-list containing all stimuli from the three possible word lists, and told that the presented words would be featured from this list. Prior to the actual transcribing of the recorded word list, a familiarization task took place in which each listener was presented with 3-4 sample words from the speaker, and was asked to transcribe the stimuli. During this trial period, listeners wore headphones and were allowed to adjust the volume of the stimuli to a comfortable listening level. Subsequently, listeners were instructed to listen to the presented word through the headphones, and type the word they heard onto the specified area on the computer screen. Word-level recordings were only presented once to each listener, as determined by the TOCS + software, and were randomized for each listener.

Ten listeners transcribed the recorded stimuli of two different speakers with DS, while four listeners transcribed stimuli from only a single speaker with DS, due to time constraints. The process of familiarization and transcription was completed by the listener for each recorded word list. Although the order of the three word lists was not controlled in their presentation to listeners judging more than one speaker's productions, individual words within each list were randomized during listener transcription. A total of three different listeners transcribed the recorded word list from each speaker with DS (as in Mahler \& Jones, 2012).

\section{Scoring and Analysis}

Scoring and error analysis of the single-word productions from the intelligibility testing was conducted from the listener transcriptions. Scoring was conducted in a "correct/incorrect" format, with each transcription compared to the original stimulus (as in Hustad, 2007). For each listener's set of responses, the transcribed words that matched the stimulus word phonemically received a "correct" score, while transcribed words that did not match the intended stimulus received an "incorrect" score. Transcription responses that did not match orthographically to the stimulus but did match phonemically (e.g., too for $t w o$ ) were scored as correct. Words marked as incorrect included errors of phoneme omission, substitution, and addition, as judged by the listeners' transcription. A final score was obtained by calculating the proportion of "correct" words from the total number of stimuli for each listener's set of responses. The three final scores obtained for each speaker, by each of the three listeners, were averaged together for the final Intelligibility Score for that speaker.

\section{Correlations}

The calculated single-word intelligibility scores were correlated with results from the acoustic analysis, in order to determine the degree to which differences in acoustic vowel space relate to and/or are predictive of overall speech intelligibility in the speakers with DS. Measures of acoustics correlated with intelligibility scores, as calculated by the cor function in R (R Core Team, 2017), included averaged results of vowel dispersions from the centroid and averaged vowel cluster ellipse size within the acoustic space.

\section{Results}

Results of the single-word intelligibility scoring collected for the participants with DS, across all three listeners, are presented below in Table. A wide range of scores was found among the speakers with DS, ranging from a lowest score of $18 \%$ to a highest score of $82 \%$. 
Table 3: Speaker intelligibility scores, listed as proportions, across all 3 listeners, and the final averaged score.

\begin{tabular}{l|lll||l}
\hline Speaker & Listener 1 & Listener 2 & Listener 3 & Total Intelligibility \\
\hline DSF5 & 0.83 & 0.78 & 0.83 & 0.82 \\
DSF7 & 0.36 & 0.36 & 0.43 & 0.38 \\
DSF8 & 0.36 & 0.34 & 0.34 & 0.35 \\
DSM2 & 0.72 & 0.73 & 0.64 & 0.70 \\
DSM3 & 0.63 & 0.63 & 0.54 & 0.60 \\
DSM9 & 0.65 & 0.64 & 0.72 & 0.67 \\
DSM10 & 0.18 & 0.19 & 0.17 & 0.18 \\
DSM11 & 0.82 & 0.73 & 0.87 & 0.81 \\
\hline \hline
\end{tabular}

Calculation of Pearson's product-moment correlation was conducted with the Stats package in R (R Core Team, 2017) for each set of variables. Intelligibility scores of the speakers with DS correlated strongest with averaged acoustic ellipse size $(r=-.85, p=.008)$, as shown in

Figure, while no correlation was found with the acoustic measure of averaged dispersion from the VFD metric $(r=.29, p=.493)$.

Figure 3: Correlation of average ellipse area in normalized $\mathrm{Hz}$ with single-word intelligibility scores for speakers with DS 


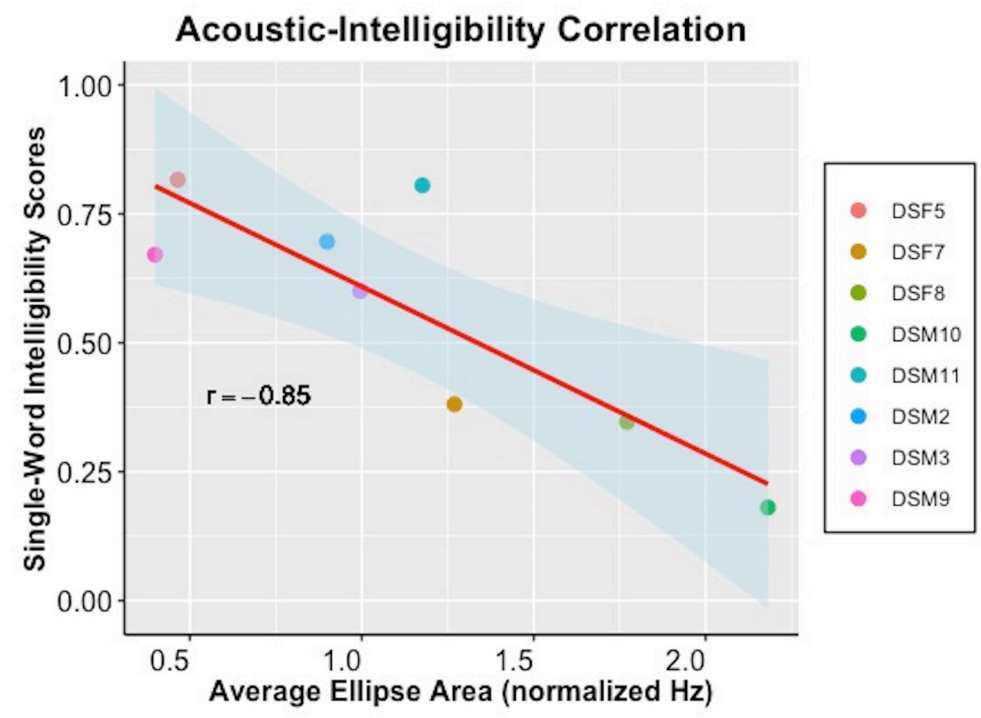

\section{General Discussion}

The following discussion addresses the acoustic and intelligibility analyses separately, followed by the associations between these domains and clinical implications. Specifically, two research questions are addressed: 1 . Do speakers with DS exhibit a reduction in the vowel acoustic space and/or increased acoustic variability of vowel production in comparison to TD peers? 2. Is single-word intelligibility correlated with acoustic vowel space in speakers with DS?

\section{Acoustics}

The results of the acoustic vowel space calculations provide insight into both the usefulness of the metrics tested and proficiency of vowel production in speakers with DS. The three vowel space metrics relying on vowel formant means in the calculations, including the Vowel Space Area (VSA), the Formant Centralization Ratio (FCR), and the DS Vocalic Anatomical Functional Ratio (DS-VR) metrics, did not demonstrate any group differences. In contrast, the Vowel Formant Dispersion (VFD) metric demonstrated group differences for the low vowels /a/ and /æ/, with TD speakers generally demonstrating larger dispersions from the weighted centroid than the speakers with DS. The difference in significant findings between the metrics may be attributed, in part, to the calculation of vowel space characteristics by vowel means for the VSA, FCR, and DS-VR metrics, without accounting for the variability of individual token production. As noted by (Karlsson \& van Doorn, 2012b), vowel space quantification based on vowel means limits the metric's efficacy in robustly discriminating between speaker groups.

The lack of differences in some of the above-noted metrics between the two speaker groups is somewhat surprising, considering the significant results found for these measures in previous studies (Bunton \& Leddy, 2011; Moura et al., 2008; O’ Leary, Lee, O’Toole, \& Gibbon, 2019), as well as the reduced intelligibility noted from the TOCS + scores in the participants with DS. One factor that differentiated the current study from Moura et al.'s (2008) is the age of participants, in that Moura et al. included children between the ages of 4 and 8, while the participants of the current study ranged from 19 to 27 years of age. Moura et al. also relied primarily, if not entirely, on the analysis of sustained vowels. The contribution of this difference is highlighted by further findings regarding intelligibility characteristics in speakers with DS (Wild et al., 2018) in which word intelligibility in general and vowel intelligibility specifically, were found to increase with age of 
the participant. If children with DS demonstrate greater reduction in speech intelligibility than TD peers in comparison to older speakers with DS, it is reasonable to assume that greater differences in vowel acoustic space may also be a characteristic of vowel production in this younger cohort. In fact, greater acoustic space differences between TD and DS groups in younger versus older speakers have been previously reported (Vorperian \& Kent, 2014). In contrast, Fourakis, Karlsson, Tilkens, and Shriberg (2010) reported a comparable positioning of high vowels /i/ and / $\mathrm{u} / \mathrm{within}$ the acoustic space (e.g., non-compressed) for speakers with DS to TD speakers, albeit shifted to lower F2 values. The current results showed a similar effect. Furthermore, Rochet-Capellan and Dohen (2015) demonstrated that the vowel space area of young adults with DS was, in fact, larger than that of TD speakers. The authors attribute this finding, in part, to increased variability of productions within the acoustic space. Finally, it is important to note that the mode of stimulus elicitation (e.g., written, pictorial, or repetition) may also affect the nature of productions in speakers with DS (Bunn, Simon, Welsh, Watson, \& Elliott, 2002).

Calculation of vowel cluster areas was also a meaningful differentiator between the speaker groups and a stronger indicator of vowel production proficiency. As reported above, speakers with DS had significantly larger areas of $95 \%$ confidence ellipses surrounding individual vowel clusters than TD speakers, across all vowels, indicating greater intra-speaker variability among participants within the DS group. Furthermore, the differentiation between the ellipse size of individual vowels, with specific reference to low vowel distinction, supports recently reported perceptual results of vowel development and accuracy in speakers with DS, in which reduced accuracy and greater variability were noted for low vowel production in comparison to TD peers (Wild et al., 2018). These results also have implications when considering production variability as a measure of overall proficiency and the impact of variable vowel production on vowel identification. Kim, Hasegawa-Johnson, and Perlman (2011) found that acoustic overlap degree between vowels served as a better discriminator between speakers with dysarthria secondary to cerebral palsy (CP) and typical controls than other acoustic space measures. The authors attribute the greater contrasts between vowels not only to a larger vowel space of corner vowels but also to reduced vowel formant variability. Although vowel space area was not a significant group predictor in the current study, the acoustic variability within vowel clusters indicates that speakers with DS demonstrate increased variability and reduced proficiency in vowel production.

Considering both the nature of low vowel production and the current results of reduced dispersion from the acoustic centroid as measured by the VFD metric, it appears that much of the variation in production of the low vowels in speakers with DS is related to impairment along the tongue-height dimension, and specifically with difficulties of tongue-jaw coordination, a critical element in production of these vowels (Johnson, Ladefoged, \& Lindau, 1993; Westbury, Lindstrom, \& McClean, 2002). In fact, this supposition provides further support for the already noted coordination deficits within overall speech production in individuals with DS, on both the phonemic (Wood, Wishart, Hardcastle, Cleland, \& Timmins, 2009) and utterance levels (Kumin, 2006). A related finding of interest was noted in the /æ/ - /i/ vowel contrast in speakers with DS, with the ellipse areas of the low vowel /æ/ being significantly larger than the ellipses of the high vowel /i/. As expected, no significant contrasts were noted between vowels for the TD speakers. These results are supported by the literature, from both perceptual and articulatory perspectives. Findings from intelligibility testing in speakers with DS across the age-span demonstrated earlier development of production accuracy, as well as overall greater accuracy for high vowels /i/ and /u/ than low vowels /a/ and /æ/ (Wild et al., 2018). From an articulatory vantage point, differences in production between high and low vowels (during perturbations) may be attributed to the 
distinctive somatosensory feedback of the tongue against the palate and/or teeth (Gick et al., 2017) that accompanies the high versus the low vowels (Mitsuya, MacDonald, Munhall, \& Purcell, 2015). Furthermore, consistent lateral lingual bracing against the upper structures of the oral cavity is noted in typical speakers for most consonants and vowels, with the exception of /a/ among the vowels (Gick et al., 2017). We would expect that /æ/, which was not explicitly discussed in Gick et al. (2017), would be similar. Although the current data confirm that typically developing speakers produce all corner vowels at comparable levels of low variability, the somatosensory feedback for high vowels may be particularly beneficial to speakers with DS, in whom the motor speech system as a whole may be impaired. In consideration of anatomical differences in speakers with DS, Wild and colleagues (2018) provide an additional rationale for differentiated intelligibility for low vowels. They suggest that reduced pharyngeal space in speakers with DS may also be a factor in impaired low vowel production, as it may contribute to a reduced articulatory working space and therefore also reduced acoustic space. While anatomical differences in speakers with DS may be a contributing factor to the acoustic results in the current study, the findings of increased variability among the low vowels and distinction in dispersion from the high vowels indicate the presence of motor speech deficits as a likely component of disordered vowel production.

\section{Acoustic-Intelligibility Correlations}

Intelligibility testing for the participants with DS was conducted in order to obtain an index of overall speech intelligibility. The current results are perhaps the first to be presented in combination with the array of acoustic metric calculations, and thus provide further insight into the nature of vowel production and its contribution to the overall speech disorder in speakers with DS. Correlations of single-word intelligibility scores with acoustic measures indicated that average vowel cluster ellipse size was strongly associated with the single word intelligibility results, such that greater ellipse size of vowel clusters in the acoustic space was predictive of reduced speech intelligibility in the speakers with DS. Increased intra-speaker variability, a commonly cited characteristic of the speech impairment in individuals with DS (Barnes et al., 2009; Hamilton, 1993; Timmins et al., 2009), may in fact be a function of immature and/or reduced control of the speech-motor system as a whole (McGowan \& McGowan, 2014; Vorperian \& Kent, 2007), and similar to the variability of acoustic patterns found in typically developing children (Ménard, Schwartz, Boë, \& Aubin, 2007). In addition to highlighting the potential contribution of vowel errors to intelligibility deficits in speakers with DS, this correlation also sheds light on the nature of the intelligibility deficit as a whole. Overall, the findings from the current study indicate that while factors such as anatomical differences and phonological disorders may affect speech production skills in speakers with DS, differences in speech motor control are a likely contributing factor to the speech impairment. These results support earlier findings of motor-speech disorder characteristics in children with DS, including features corresponding to combined symptoms of both dysarthria and childhood apraxia of speech (Rupela et al., 2016; Wilson et al., 2019), evidence of temporal variability in speech production (Brown-Sweeney \& Smith, 1997), and impairments of motor coordination and strength (Zarzo-Benlloch, Cervera-Mérida, \& Ygual-Fernández, 2017). The precise differentiation between the motor impairments of dysarthria or apraxia of speech is difficult to ascertain from the current data, as vowel impairment and a reduced acoustic vowel space are noted in both disorders (e.g., den Ouden, Galkina, Basilakos, \& Fridriksson, 2018; Levy et al., 2016).

\section{Limitations and Future Directions}


The findings of the current study are a further contribution to the understanding of speech production and intelligibility deficits in speakers with DS, but several limitations warrant further investigations. Most notably, the small number of participants on which the analyses were conducted inherently limit the generalization of these results to the population at large and may explain some inconsistencies between current results and previous findings. Additionally, further investigations would benefit from inclusion of characteristics relating to motor speech impairment, language skills, and cognitive skills of participants with DS; such information was not available in the current study. Although intelligibility is reported to be poorly correlated with language or cognitive skills in individuals with DS (Cleland, Wood, Hardcastle, Wishart, \& Timmins, 2010), information regarding these domains would complement speech production and intelligibility findings. Hearing loss was not considered a confounding factor in the present study, as impairment to hearing or auditory structures is a prevalent characteristic of the syndrome. As conducted in previous studies (Wild et al., 2018), mild hearing impairment did not exclude participants with DS in the current study, inasmuch as no significant hearing loss or use of hearing devices were reported. In fact, one of the participants (DSF5) who did not pass the hearing screening received the highest intelligibility score. Exceptions notwithstanding, a larger cohort of participants in future studies would allow for increased control of hearing characteristics and impairment. A final limitation of the current study is noted in the absence of an error analysis for the intelligibility testing. Ideally, a thorough analysis of such intelligibility errors should be obtained from closedset, phonemic contrast judgements, and is a noteworthy topic for future research.

\section{Clinical Implications}

The current findings have several clinical implications for the assessment and treatment of speech production deficits in speakers with DS. Perhaps most importantly, these results provide insight into the nature of disorder, which, in turn, can help guide decisions regarding intervention approaches and treatment targets for increasing speech intelligibility. As noted above, the potential contribution of motor control deficits to the reduced intelligibility support the use of a motor-based approach in assessment and treatment of speech production deficits, with a focus on lingual precision and control, inter-articulatory coordination, and consistency of productions. Motorbased approaches for the treatment of motor speech disorders have been previously studied and used effectively in the treatment literature, with applications for the treatment of both apraxia of speech (e.g., Maas, Butalla, \& Farinella, 2012; Strand \& Debertine, 2000) and dysarthria (e.g., Levy, 2014; Levy, Ramig, \& Camarata, 2013; Park, Theodoros, Finch, \& Cardell, 2016; Sapir, Spielman, Ramig, Story, \& Fox, 2007). Although the specific treatment regimen may differ depending on the characteristics of the disorder and target population, they all incorporate principles designed to increase and enhance the learning of the motor skill, also called Principles of Motor Learning (see Maas et al., 2008; Schmidt, 1975). The current research does not aim to provide a means of distinguishing between the motor speech disorders of dysarthria and apraxia, partially in light of the difficulty in making this distinction in individuals with DS, who may present with many speech characteristics that are potentially overlapping between the two disorders (Rupela et al., 2016; Wilson et al., 2019). Although beyond the scope of this study, the research literature would benefit from further work to address the differentiation of these disorders in individuals with DS, and the therapeutic implications thereafter. Nonetheless, the results presented above provide general guidelines for treatment, with specific consideration of potentially impaired vowel production in this population.

Although vowels are not a common target in traditional articulation treatment approaches (Gibbon, 2013), they may, in fact, be important therapeutically, both in terms of their impact on 
the perceptual accuracy of the speech signal (Lansford \& Liss, 2014a) and in light of the potential increase of lingual control on a broad level, a skill necessary for accurate speech production (Wild et al., 2018). It should also be noted that certain speech interventions, including clear speech (Lam, Tjaden, \& Wilding, 2012; Levy et al., 2017; Park et al., 2016) and prosody production (Connaghan \& Patel, 2017) facilitate improved vowel distinctiveness, even without directly treating vowels as a therapeutic target. The efficacy of treatments for articulation and/or intelligibility impairments in adolescents and adults with DS is particularly limited in the therapeutic literature. However, reports of speech gains following behavioral and instrumental interventions in adults with DS (Kent \& Vorperian, in press; Mahler \& Jones, 2012) as well as documentation of naturally increasing speech intelligibility through 16 years of age (Wild et al., 2018) both implicate potential gains in this population past childhood. Further study is warranted to determine how vowelbased and/or motor-based speech treatments impact intelligibility skills, as well as the efficacy of all interventions in speakers with DS both within and beyond the period of developmental change.

Acknowledgements: This research was funded in part by the Doctoral Student Research Grant, awarded by The Graduate Center, CUNY. We thank two anonymous reviewers and the editor for helpful comments.

\section{References:}

Adank, P., Smits, R., \& van Hout, R. (2004). A comparison of vowel normalization procedures for language variation research. The Journal of the Acoustical Society of America, 116(5), 3099-3107.

Baayen, R. H., Davidson, D. J., \& Bates, D. M. (2008). Mixed-effects modeling with crossed random effects for subjects and items. Journal of Memory and Language, 59(4), 390-412.

Barnes, E., Roberts, J., Long, S. H., Martin, G. E., Berni, M. C., Mandulak, K. C., \& Sideris, J. (2009). Phonological accuracy and intelligibility in connected speech of boys with fragile $\mathrm{X}$ syndrome or Down syndrome. Journal of Speech, Language, and Hearing Research, 52(4), $1048-61$.

Bates, D., Maechler, M., Bolker, B., \& Walker, S. (2015). Fitting linear mixed-effects models using lme4. Journal of Statistical Software, 67(1), 1-48.

Bernardi, G. F., Pires, C. T. F., Oliveira, N. P., \& Nisihara, R. (2017). Prevalence of pressure equalization tube placement and hearing loss in children with Down syndrome. International Journal of Pediatric Otorhinolaryngology, 98, 48-52.

Boersma, P., \& Weenink, D. (2017). Praat: Doing phonetics by computer (Version 6.0.28).

Bradlow, A., Torretta, G. M., \& Pisoni, D. B. (1996). Intelligibility of normal speech I: Global and fine-grained acoustic-phonetic talker characteristics. Speech Communication, 20, 255272.

Brown-Sweeney, S., \& Smith, B. (1997). The development of speech production abilities in children with Down syndrome. Clinical Linguistics \& Phonetics, 11(5), 345-362.

Bunn, L., Simon, D. a, Welsh, T. N., Watson, C., \& Elliott, D. (2002). Speech production errors in adults with and without Down syndrome following verbal, written, and pictorial cues. Developmental Neuropsychology, 21(2), 157-72.

Bunton, K., \& Leddy, M. (2011). An evaluation of articulatory working space area in vowel production of adults with Down syndrome. Clinical Linguistics \& Phonetics, 25(4), 321-34.

Bunton, K., Leddy, M., \& Miller, J. (2009). Phonetic intelligibility testing in adults with Down syndrome. Down Syndrome Research and Practice, 12(1), 1-4.

Carl, M. (2018). Vowel Production in Down Syndrome - An Ultrasound Study. (Unpublished 
doctoral dissertation). The Graduate Center, City University of New York. New York, NY.

Chiba, T., \& Kajiyama, M. (1941). The vowel: Its nature and structure. Tokyo: Tokyo-Kaiseikan Publishing Co., Ltd.

Cleland, J., Wood, S., Hardcastle, W., Wishart, J., \& Timmins, C. (2010). Relationship between speech, oromotor, language and cognitive abilities in children with Down's syndrome.

International Journal of Language \& Communication Disorders, 45(1), 83-95.

Connaghan, K. P., \& Patel, R. (2017). The impact of contrastive stress on vowel acoustics and intelligibility in dysarthria. Journal of Speech, Language, and Hearing Research, 60(1), 3850.

den Ouden, D.-B., Galkina, E., Basilakos, A., \& Fridriksson, J. (2018). Vowel formant dispersion reflects severity of apraxia of speech. Aphasiology, 32(8), 902-921.

Desai, S. S. (1997). Down syndrome: A review of the literature. Oral Surgery, Oral Medicine, Oral Pathology, Oral Radiology, and Endodontics, 84(3), 279-85.

Fourakis, M., Karlsson, H., Tilkens, C., \& Shriberg, L. (2010). Acoustic correlates of nasopharyngeal resonance. In A. Botinis (Ed.), Proceedings of the 3rd ISCA Tutorial and Research Workshop on Experimental Linguistics (pp. 41-44). Athens, Greece: University of Athens and the International Speech Communication Association (ISCA).

Gibbon, F. E. (2013). Therapy for children with abnormal vowels. In M. J. Ball \& F. E. Gibbon (Eds.), Handbook of vowels and vowel disorders (pp. 429-446). New York, NY: Psychology Press.

Gick, B., Allen, B., Roewer-Després, F., \& Stavness, I. (2017). Speaking tongues are actively braced. Journal of Speech Language and Hearing Research, 60(3), 494-506.

Hamilton, C. (1993). Investigation of the articulatory patterns of young adults with Down's syndrome using electropalatography. Down Syndrome Research and Practice, 1(1), 15-28.

Hillenbrand, J. M., Clark, M. J., \& Nearey, T. M. (2001). Effects of consonant environment on vowel formant patterns. Journal of the Acoustical Society of America, 109(2), 748-763.

Hodge, M., Daniels, J., \& Gotzke, C. L. (2009). TOCS+ Intelligibility Measures (Version 5.3) [Computer software]. Edmonton, Canada: University of Alberta.

Hodge, M., \& Gotzke, C. (2010). Stability of intelligibility measures for children with dysarthria and Cerebral Palsy. Journal of Medical Speech-Language Pathology, 18(4), 61-65.

Hodge, M., \& Gotzke, C. L. (2014). Construct-related validity of the TOCS measures: Comparison of intelligibility and speaking rate scores in children with and without speech disorders. Journal of Communication Disorders, 51, 51-63.

Hustad, K. C. (2007). Effects of speech stimuli and dysarthria severity on intelligibility scores and listener confidence ratings for speakers with cerebral palsy. Folia Phoniatrica et Logopaedica, 59, 306-317.

Hustad, K. C., Gorton, K., \& Lee, J. (2010). Classification of speech and language profiles in 4year-old children with cerebral palsy: a prospective preliminary study. Journal of Speech, Language, and Hearing Research: JSLHR, 53(6), 1496-513.

Johnson, K., Ladefoged, P., \& Lindau, M. (1993). Individual differences in vowel production. The Journal of the Acoustical Society of America, 94(2), 701-714.

Jones, H. N., Crisp, K. D., Kuchibhatla, M., Mahler, L., Risoli, T., Jones, C. W., \& Kishnani, P. (2019). Auditory-perceptual speech features in children with down syndrome. American Journal on Intellectual and Developmental Disabilities, 124(4), 324-338.

Karlsson, F., \& van Doorn, J. (2012a). Applying the Vowel Formant Dispersion (VFD) method to the study of reduced or alterered vowel productions. In Poster presented at the 14th 
Meeting of the International Clinical Phonetics and Linguistics Association. Cork, Ireland. Karlsson, F., \& van Doorn, J. (2012b). Vowel formant dispersion as a measure of articulation proficiency. The Journal of the Acoustical Society of America, 132(4), 2633-41.

Kent, R. D., \& Kim, Y. J. (2003). Toward an acoustic typology of motor speech disorders. Clinical Linguistics \& Phonetics, 17(6), 427-45.

Kent, R. D., \& Vorperian, H. K. (n.d.). Speech intelligibility and communication in Down syndrome: Intervention approaches and the role of speech supplementation across the lifespan. In T. appear in K. Wilkinson \& L. H. Finestack (Eds.), Multi-Modal AAC for Individuals with Down Syndrome across the Lifespan. Baltimore: Brookes Publishing.

Kent, R. D., \& Vorperian, H. K. (2013). Speech impairment in Down syndrome: A review. Journal of Speech, Language, and Hearing Research, 56(1), 178-210.

Kent, R. D., \& Vorperian, H. K. (2018). Static measurements of vowel formant frequencies and bandwidths: A review. Journal of Communication Disorders, 74, 74-97.

Kim, H., Hasegawa-Johnson, M., \& Perlman, A. (2011). Vowel contrast and speech intelligibility in dysarthria. Folia Phoniatrica et Logopaedica, 63(4), 187-94.

Kumin, L. (1994). Intelligibility of speech in children with Down syndrome in natural settings parents' perspective. Perceptual and Motor Skills, 78, 307-313.

Kumin, L. (2006). Speech intelligibility and childhood verbal apraxia in children with Down syndrome. Down Syndrome Research and Practice, 10(1), 10-22.

Lam, J., Tjaden, K., \& Wilding, G. (2012). Acoustics of clear speech: Effect of instruction. Journal of Speech Language and Hearing Research, 55, 1807-1822.

Lammert, A., Proctor, M. I., \& Narayanan, S. S. (2013). Interspeaker variability in hard palate morphology and vowel production. Journal of Speech, Language, and Hearing Research, 56, S1924-S1933.

Lansford, K., \& Liss, J. (2014a). Vowel acoustics in dysarthria: Mapping to perception. Journal of Speech, Language, and Hearing Research, 57(February), 68-80.

Lansford, K., \& Liss, J. (2014b). Vowel acoustics in dysarthria: Speech disorder diagnosis and classification. Journal of Speech, Language, and Hearing Research, 57, 57-67.

Lenth, R. V. (2016). Least-squares means: The R package lsmeans. Journal of Statistical Software, 69(1).

Levy, E. (2014). Implementing two treatment approaches to childhood dysarthria. International Journal of Speech-Language Pathology, 16(4), 344-354.

Levy, E., Chang, Y. M., Ancelle, J. A., \& McAuliffe, M. J. (2017). Acoustic and perceptual consequences of speech cues for children with dysarthria. Journal of Speech Language and Hearing Research, 60, 1766-1779.

Levy, E., Leone, D., Moya-Galé, G., Hsu, S., Chen, W., \& Ramig, L. O. (2016). Vowel intelligibility in children with and without dysarthria: An exploratory study. Communication Disorders Quarterly, 37(3), 171-179.

Levy, E., Ramig, L. O., \& Camarata, S. M. (2013). The effects of two speech interventions on speech function in pediatric dysarthria. Journal of Medical Speech-Language Pathology, 20(4), 82-87.

Lobanov, B. M. (1971). Classification of Russian vowels spoken by different speakers. The Journal of the Acoustical Society of America, 49(2B), 606-608.

Maas, E., Butalla, C. E., \& Farinella, K. A. (2012). Feedback frequency in treatment for childhood apraxia of speech. American Journal of Speech-Language Pathology, 21, 239 257. 
Maas, E., Robin, D. A., Hula, S. N. A., Freedman, S. E., Wulf, G., Ballard, K. J., \& Schmidt, R. A. (2008). Principles of motor learning in treatment of motor speech disorders. American Journal of Speech-Language Pathology, 17, 277-298.

Mahler, L. A., \& Jones, H. N. (2012). Intensive treatment of dysarthria in two adults with Down syndrome. Developmental Neurorehabilitation, 15(1), 44-53.

Mai, C. T., Kucik, J. E., Isenburg, J., Feldkamp, M. L., Marengo, L. K., Bugenske, E. M., ... Kirby, R. S. (2013). Selected birth defects data from population-based birth defects surveillance programs in the United States, 2006 to 2010: featuring trisomy conditions. Birth Defects Research. Part A, Clinical and Molecular Teratology, 97(11), 709-25.

McCloy, D. R. (2016). phonR: Tools for phoneticians and phonologists.R package version 1.0-7. https://cran.r-project.org/web/packages/phonR/phonR.pdf.

McCloy, D. R. (2017). Vowel ellipse overlap. Retrieved from https://gist.github.com/drammock/36fc241fe31378f1 ecc9\#file-vowel-ellipse-overlap-r-L1L31

McCloy, D. R., Wright, R., \& Souza, P. (2014). Modeling intrinsic intelligibility variation: vowel-space size and structure, 18, 060007.

McGowan, R., \& McGowan, R. (2014). A longitudinal study of very young children's vowel production. Journal of Speech, Language and Hearing Research, 57(February), 1-15.

Ménard, L., Schwartz, J. L., Boë, L. J., \& Aubin, J. (2007). Articulatory-acoustic relationships during vocal tract growth for French vowels: Analysis of real data and simulations with an articulatory model. Journal of Phonetics, 35(1), 1-19.

Mitsuya, T., MacDonald, E. N., Munhall, K. G., \& Purcell, D. W. (2015). Formant compensation for auditory feedback with English vowels. The Journal of the Acoustical Society of America, 138(1), 413-424.

Moura, C. P., Cunha, L. M., Vilarinho, H., Cunha, M. J., Freitas, D., Palha, M., ... PaisClemente, M. (2008). Voice parameters in children with Down syndrome. Journal of Voice, $22(1), 34-42$.

Neel, A. T. (2008). Vowel space characteristics and vowel identification accuracy. Journal of Speech Language and Hearing Research, 51(3), 574-585.

O' Leary, D., Lee, A., O'Toole, C., \& Gibbon, F. (2019). Perceptual and acoustic evaluation of speech production in Down syndrome: A case series. Clinical Linguistics \& Phonetics, $0(0)$, $1-20$.

Park, S., Theodoros, D., Finch, E., \& Cardell, E. (2016). Be Clear: A new intensive speech treatment for adults with nonprogressive dysarthria. American Journal of Speech-Language Pathology, 25, 97-100.

Peterson, G., \& Barney, H. (1952). Control methods used in the study of vowels. The Jarnal of the Acoustical Society of America, 24(2), 175-184.

R Core Team. (2017). R: A language and environment for statistical computing. Vienna, Austria: R Foundation for Statistical Computing. URL https://www.r-project.org/.

Rochet-Capellan, A., \& Dohen, M. (2015). Acoustic characterisation of vowel production by young adults with Down syndrome. In 18th International Congress of Phonetic Sciences (ICPhS) (pp. 1-5).

Rupela, V., Velleman, S. L., \& Andrianopoulos, M. V. (2016). Motor speech skills in children with Down syndrome: A descriptive study. International Journal of Speech-Language Pathology, 18(5), 483-492.

Sapir, S., Ramig, L. O., Spielman, J. L., \& Fox, C. (2010). Formant centralization ratio : A 
proposal for a new acoustic measure of dysarthric speech. Journal of Speech, Language, and Hearing Research, 53, 114-125.

Sapir, S., Spielman, J. L., Ramig, L. O., Story, B. H., \& Fox, C. (2007). Effects of intensive voice treatment (the Lee Silverman Voice Treatment [LSVT]) on vowel articulation in dysarthric individuals with idiopathic Parkinson disease: acoustic and perceptual findings. Journal of Speech, Language, and Hearing Research, 50(4), 899-912.

Schmidt, R. A. (1975). PSYCHOLOGICAL REVIEW, 82(4).

Skodda, S., Visser, W., \& Schlegel, U. (2011). Vowel articulation in Parkinson's disease. Journal of Voice, 25(4), 467-72.

Strand, E., \& Debertine, P. (2000). Efficacy of integral stimulation intervention with developmental apraxia of speech. Journal of Medical Speech-Language Pathology, 8(4), 295-300.

Strange, W., Weber, A., Levy, E. S., Shafiro, V., Hisagi, M., \& Nishi, K. (2007). Acoustic variability within and across German, French, and American English vowels: Phonetic context effects. The Journal of the Acoustical Society of America, 122(2), 1111-1129.

Timmins, C., Cleland, J., Wood, S. E., Hardcastle, W. J., \& Wishart, J. G. (2009). A perceptual and electropalatographic study of $/ \mathrm{g} /$ in young people with Down's syndrome. Clinical Linguistics \& Phonetics, 23(12), 911-25.

Tjaden, K., Lam, J., \& Wilding, G. (2013). Vowel acoustics in Parkinson's disease and Multiple Sclerosis: Comparison of clear, loud, and slow speaking conditions. Journal of Speech, Language, and Hearing Research, 56, 1485-1502.

Tjaden, K., \& Wilding, G. E. (2005). Effect of Rate Reduction and Increased Loudness on Acoustic Measures of Anticipatory Coarticulation in Multiple Sclerosis and Parkinson's Disease. Journal of Speech, Language, and Hearing Research, 48, 261-277.

Vorperian, H. K., \& Kent, R. D. (2007). Vowel Acoustic Space Development in children: A synthesis of acoustic and Anatomic Data. Journal of Speech, Language, and Hearing Research, 50, 1510-1545.

Vorperian, H. K., \& Kent, R. D. (2014). Development of the Acoustic Vowel Quadrilateral: Normative Data and a Clinical Application. Poster presented at the Motor Speech Conference, Sarasota, Fl.

Weismer, G., Jeng, J. Y., Laures, J. S., Kent, R. D., \& Kent, J. F. (2001). Acoustic and intelligibillity characteristics of sentence production in neurogenic speech disorders. Folia Phoniatrica et Logopaedica, 53, 1-18.

Westbury, J. R., Lindstrom, M. J., \& McClean, M. D. (2002). Tongues and lips without jaws: a comparison of methods for decoupling speech movements. Journal of Speech, Language, and Hearing Research, 45, 651-662.

Wild, A., Vorperian, H. K., Kent, R., Bolt, D. M., \& Austin, D. (2018). Single-word speech intelligibility in children and adults with Down syndrome. American Journal of SpeechLanguage Pathology, 27, 1-15222-236.

Wilson, E. M., Abbeduto, L., Camarata, S. M., \& Shriberg, L. D. (2019). Estimates of the prevalence of speech and motor speech disorders in adolescents with Down syndrome. Clinical Linguistics and Phonetics, 33(8), 772-789.

Wood, S., Wishart, J., Hardcastle, W., Cleland, J., \& Timmins, C. (2009). The use of electropalatography (EPG) in the assessment and treatment of motor speech disorders in children with Down's syndrome: evidence from two case studies. Developmental Neurorehabilitation, 12(2), 66-75. 
Yang, J., \& Fox, R. A. (2013). Acoustic development of vowel production in American English children. Proceedings of the Annual Conference of the International Speech Communication Association, INTERSPEECH, (April), 1263-1267.

Yunusova, Y., Weismer, G., Kent, R. D., \& Rusche, N. M. (2005). Breath-group intelligibility in dysarthria: characteristics and underlying correlates. Journal of Speech, Language, and Hearing Research, 48(6), 1294-1310.

Zarzo-Benlloch, M., Cervera-Mérida, J. F., \& Ygual-Fernández, A. (2017). Variables that influence articulation accuracy in children with Down Syndrome and Specific Language Disorder: Similarities and differences. Advances in Speech-Language Pathology, Fernanda Dreux M. Fernandes, IntechOpen, DOI: 10.5772/intechopen.69933. Available from: https://www.intechopen.com/books/advances-in-speech-language-pathology/variables-thatinfluence-articulation-accuracy-in-children-with-down-syndrome-and-specific-language-, 199-217. 